\title{
Utility of the 'Fuzzy Area' for Active Myopic Choroidal Neovascularization Detection by Spectral-Domain Optical Coherence Tomography
}

\section{Giuseppe Casalino Ugo Introini Giuseppe Querques Francesco Bandello}

Department of Ophthalmology, San Raffaele Scientific Institute, Vita-Salute University, Milan, Italy

We read with interest the article by Milani et al. [1] entitled 'Imaging of naive myopic choroidal neovascularization by spectral-domain optical coherence tomography'. We congratulate the authors for giving new insights into the appearance of myopic choroidal neovascularization (CNV) on spectral-domain optical coherence tomography (SD-OCT).

Active myopic CNV usually shows weak leakage on fluorescein angiography (FA) and subtle retinal changes on optical coherence tomography (OCT), and as a result the diagnosis can be a challenge. FA and SD-OCT do not seem to have a good agreement in detecting myopic $\mathrm{CNV}$, and FA is still considered the most valuable tool for detecting myopic CNV activity $[2,3]$.
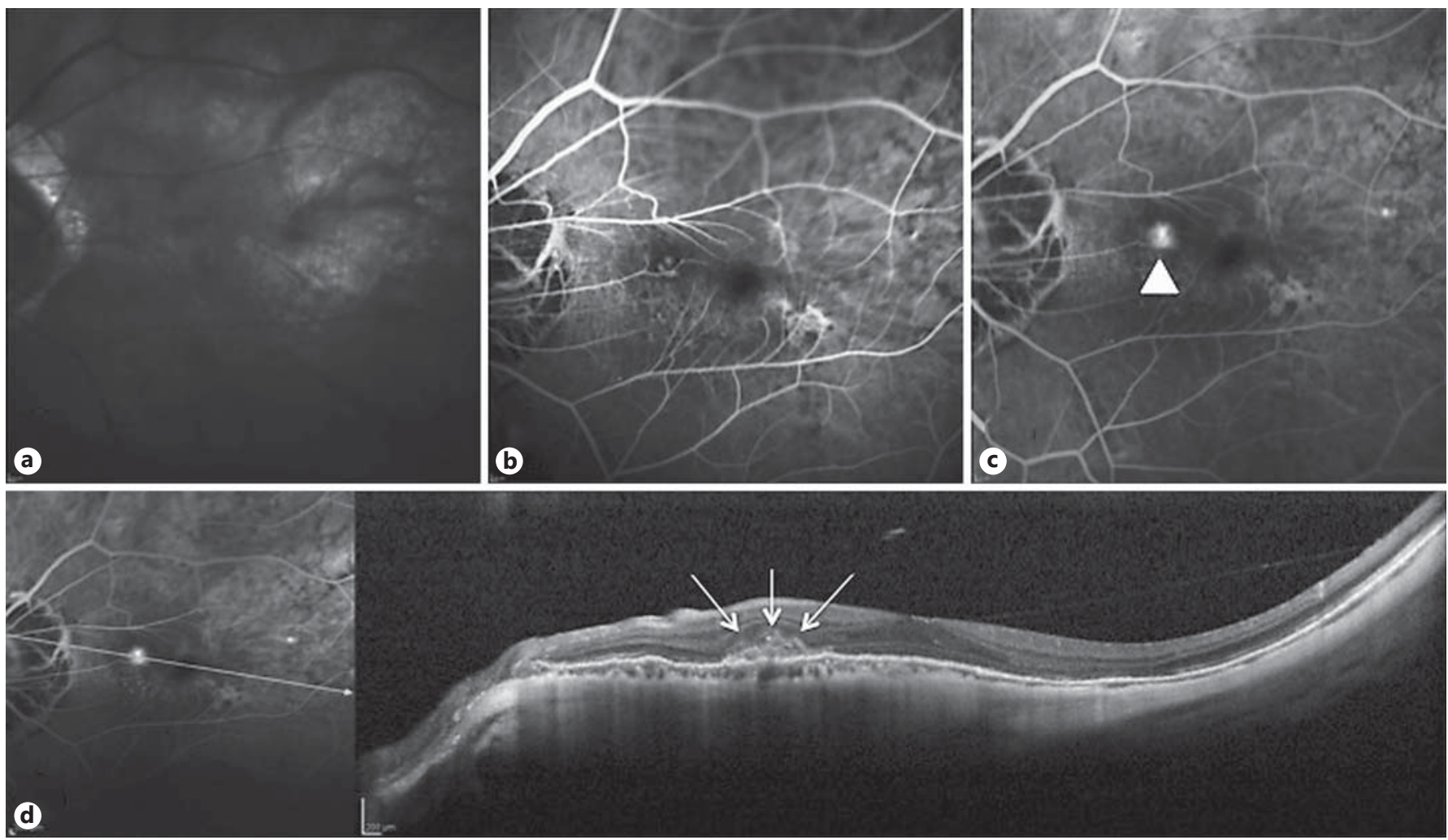

Fig. 1. Infrared imaging (a) and early (b) and late phase (c) of FA with SD-OCT (d) in a naïve myopic CNV. Late phase of FA (c) shows fluorescein dye leakage (arrowhead) and SD-OCT scan passing through the leaking lesion shows a hyperreflective lesion with fuzzy borders ('fuzzy area', arrows), consistent with the active myopic CNV.

\section{KARGER 125}

2014 S. Karger AG, Base

$0030-3755 / 14 / 2331-0056 \$ 39.50 / 0$

E-Mail karger@karger.com

www.karger.com/oph 

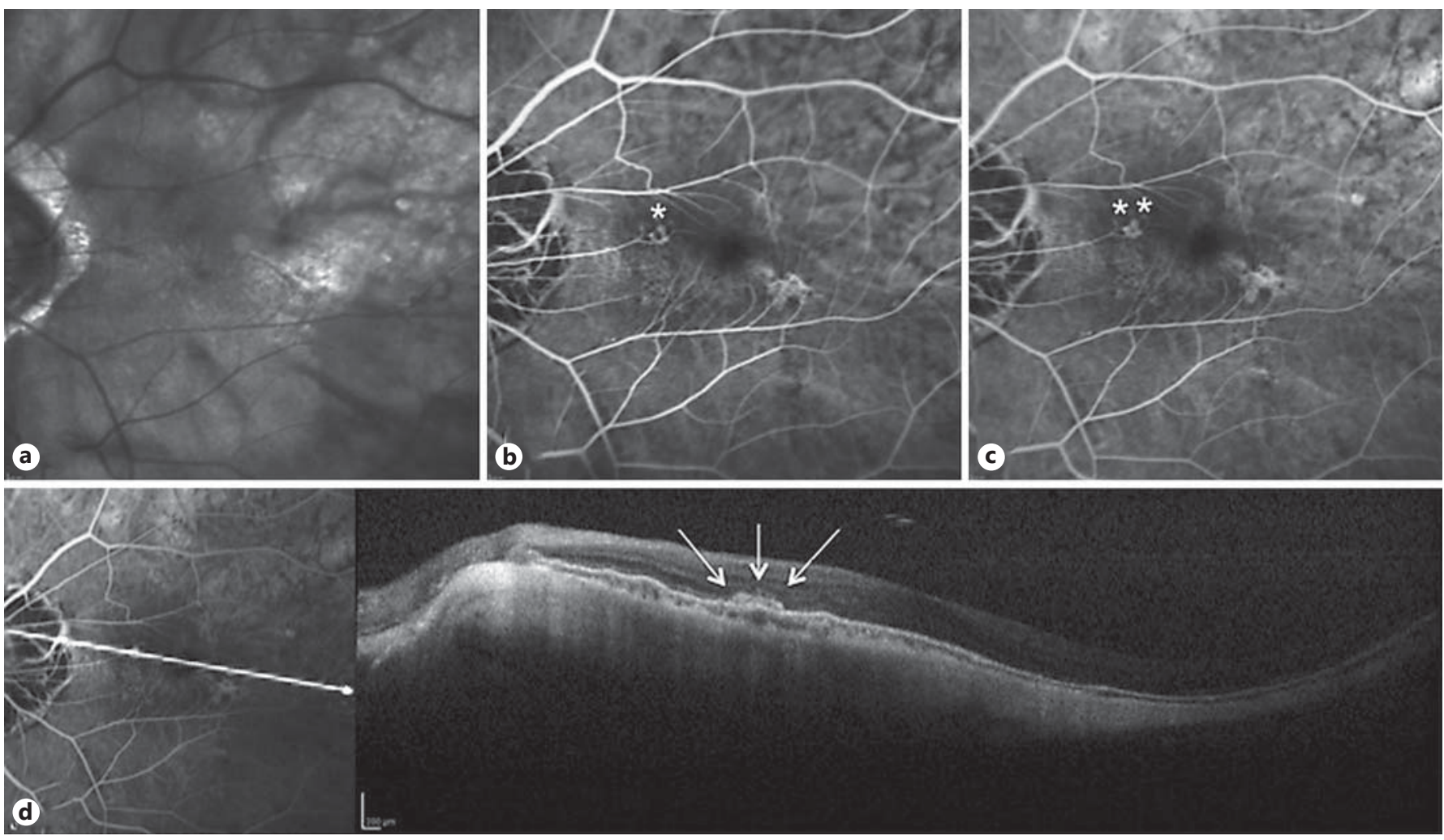

Fig. 2. Infrared imaging (a) and early (b) and late phase (c) of FA with SD-OCT (d) 1 month following anti-VEGF treatment for myopic CNV. Late phase of FA (c) shows fluorescein dye staining with no leakage (asterisks) and SD-OCT scan passing through the lesion shows slight thickening of RPE (arrows) consistent with an inactive myopic CNV.

In 2012, our team showed an SD-OCT hyperreflective lesion with fuzzy borders ('fuzzy area') and a more highly reflective core above the retinal pigment epithelium (RPE) associated with thickening of the overlying retina in eyes with naïve myopic CNVs [4]. In our series, the presence of the 'fuzzy area' highly agreed with FA dye leakage and we suggested that this fuzzy aspect on SD-OCT could be caused by the outer retinal swelling associated with myopic active neovascular proliferation. Of note, the disappearance of the 'fuzzy area', along with thickening of the RPE, agreed with the absence of FA dye leakage following antiVEGF treatment [4].

As pointed out by the authors, in our series we used SD-OCT with a lower resolution (Spectral OCT/SLO; Opko/OTI, Miami, Fla., USA) compared to the SD-OCT used in their study (Spectralis; Heidelberg Engineering, Heidelberg, Germany). This could explain why we did not investigate the different SD-OCT features of the neu- roretinal layers associated with myopic $\mathrm{CNV}$, which were instead reported in the authors' paper [1]. On the other hand, we wonder whether these features (i.e. outer and inner nuclear layer changes and internal and external plexiform layer changes) could help clinicians in detecting myopic CNV activity. In light of this, we would like to ask the authors the utility, in clinical practice, of investigating SD-OCT features other than the 'fuzzy area' in order to evaluate myopic CNV activity and, possibly, the response to the treatment. As far as this is concerned, we report one representative naïve myopic $\mathrm{CNV}$ which was detected before and after treatment with high-resolution SD-OCT at our department (fig. 1,2).

\section{Disclosure Statement}

The authors have no conflicts of interest to declare.

\section{References}

1 Milani P, Pece A, Pierro L, Bergamini F: Imaging of naive myopic choroidal neovascularization by spectral-domain optical coherence tomography. Ophthalmologica 2014;232:2836.

2 Leveziel N, Caillaux V, Bastuji-Garin S, Zmuda M, Souied EH: Angiographic and optical coherence tomography characteristics of recent myopic choroidal neovascularization. Am J Ophthalmol 2013;155:913-919.

- 3 Iacono P, Battaglia Parodi M, Papayannis A, Kontadakis S, Da Pozzo S, Cascavilla ML, La Spina C, Varano M, Bandello F: Fluorescein angiography and spectral-domain optical coherence tomography for monitoring antiVEGF therapy in myopic choroidal neovascularization. Ophthalmic Res 2014;52:25-31.

4 Introini U, Casalino G, Querques G, Gimeno AT, Scotti F, Bandello F: Spectral-domain OCT in anti-VEGF treatment of myopic choroidal neovascularization. Eye (Lond) 2012; 26:976-982. 